Herausgegeben

im Auftrage des Ministerpräsidenten Dr. Franz Meyers

von Staatssekretär Professor Dr.h.c. Dr. E.h. Leo Brandt

DK 308:631 (55)

$338: 631(55)$

$308(55-77)$

Nr. 1021

Forschungsinstitut für Internationale Technische Zusammenarbeit an der Rheinisch-Westfälischen Technischen Hochschule Aachen

(FIZ)

Dr. agr. Ulrich Planck

Institut für Agrarpolitik und Sozialökonomik des Landbaus an der Landwirtschaftlichen Hochschule Stuttgart-Hohenheim

\title{
Die sozialen und ökonomischen Verhältnisse in einem iranischen Dorf
}

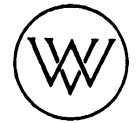

WESTDEUTSCHER VERLAG / KOLNUNDOPLADEN 
ISBN 978-3-663-03473-5

DOI 10.1007/978-3-663-04662-2

ISBN 978-3-663-04662-2 (eBook) 
Das Forschungsinstitut für internationale technische Zusammenarbeit hat die vorliegende Arbeit bewußt in die Reihe seiner Veröffentlichungen aufgenommen, da wissenschaftliche Untersuchungsergebnisse über die sozialen und ökonomischen Verhältnisse in den Entwicklungsländern die wesentlichen Voraussetzungen für die später einsetzende technische Hilfe und Zusammenarbeit bilden.

$\mathrm{Zu}$ diesen notwendigen Informationen gehört auch das wissen um die geistige Einstellung der Menschen sowie die Kenntnis der wirtschaftlichen und sozialen Verhältnisse in allen Bereichen, die für eine Erhöhung des Sozialprodukts in Betracht kommen.

Der Verfasser der vorliegenden Schrift hat es unternommen, für den Iran am Beispiel von zwei ausgewählten Dörfern eingehende Untersuchungen durchzuführen, aus denen er die Möglichkeiten einer sinnvollen Förderung dieses ausbaufähigen Landes ableitet.

Prof. Dr.-Ing. Dr.-Ing. E.h. Hermann Schenck Direktor des Forschungsinstituts 
Bei der Beschäftigung mit den agrarökonomischen und agrarsozialen Problemen der Entwicklungsländer muB man immer wieder feststellen, dab unsere Kenntnis von den Agrarzuständen in diesen Ländern unzulänglich ist. In der Regel gibt es für eine einwandfreie Darstellung solcher Fakten nicht genügend wissenschaftiche und statistische Unterlagen. So ist man z.B. in bezug auf eines der wichtigsten Kennzeichen der Agrarstruktur, nämlich der durchschnittlichen Größe der landwirtschaftlichen Betriebe, bisher im wesentlichen auf grobe Schätzungen angewiesen. Es gibt statistische Daten im allgemeinen nur über die Grundeigentumsverhältnisse, und dies auch nur dort, wo Grundbücher geführt werden. Dagegen lagen Angaben über die Zahl und Größe der landwirtschaftlichen Betriebe in der Regel nicht vor, bis im Jahre 1960 die von der FAO in Gang gebrachte internationale Erhebung durchgeführt wurde, deren Ergebnisse noch nicht bekanntgegeben sind. Aber auch für andere wichtige Merkmale der Agrarstruktur sind bisher die Angaben der Statistik zum großen Teil unzureichend und unzuverlässig.

Unter diesen Umständen sind monographische Dorfuntersuchungen eines der wichtigsten Mittel, um uns eine bessere Kenntnis von den Agrarzuständen in den Entwicklungsländern zu verschaffen. Eine bessere Kenntnis ist aber eine wesentliche Voraussetzung für eine zweckentsprechende Planung der landwirtschaftlichen Entwicklungsprogramme und der Entwicklungshilfe. Es kommt hinzu, daB in den kleinbäuerlichen Betrieben, die keine Buchführung haben, manche wichtigen Daten nicht durch die Statistik erfabt werden können, sondern sich nur an einigermaßen repräsentativen Einzelbeispielen illustrieren lassen. Auch in der Bundesrepublik ist z.B. für die Feststellung der Ertragslage der kleinbäuerlichen Betriebe die monographische Dorfuntersuchung ein wichtiges Hilfsmittel.

Als ich im Frühjahr 1960 Gelegenheit hatte, bei einem Aufenthalt im Iran fachliche Kontakte aufzunehmen, habe ich bei den dortigen Stellen angeregt, systematisch sozialökonomische Dorfuntersuchungen in Gang zu bringen. Ich habe angeboten, daB Herr Dr. Ulrich PLANCK, Assistent an dem damals noch von mir geleiteten Institut für Agrarpolitik und Sozialökonomik des Landbaus in Stuttgart-Hohenheim, mit einem Forschungsauftrag nach dem Iran kommen könnte, um in einem geeigneten Dorf eine solche monographische Studie durchzuführen und damit auch die in Deutschland bewährte Methodik derartiger Untersuchungen im Iran zu demonstrie- 
ren. Ich konnte darauf hinweisen, daB ich selbst zu sehr aufschlubreichen Ergebnissen gekommen bin, als ich seinerzeit, während meiner Tätigkeit für die FAO, derartige Dorfuntersuchungen in Pakistan durchzuführen hatte.

Da die iranischen Regierungsstellen für das Forschungsvorhaben großes Interesse zeigten und ihre volle Unterstütung zusagten, kam der Forschungsauftrag noch im gleichen Jahre zustande. Herr Dr. PLANCK hatte sich schon dadurch in die Materie eingearbeitet, daB er mich zwei Jahre lang bei der Anleitung eines iranischen Studierenden zu unterstützen hatte, der in dem genannten Hohenheimer Institut eine Dissertation über die Agrarverfassung des Iran angefertigt hat. Dr.PLANCK hat die Untersuchung im Herbst 1960 während eines dreimonatigen Aufenthalts im Iran durchgefürt, wofür ihm von der Deutschen Forschungsgemeinschaft dankenswerterweise eine Reisebeihilfe gegeben wurde.

Das Forschungsvorhaben wurde durch das Landwirtschaftsministerium in Teheran unterstütz, das auch eine jüngere iranische Fachkraft, Herrn SHAKERI, als Mitarbeiter und Dolmetscher zur Verfügung stellte. Er hat sich durch seine kameradschaftliche Hilfe bei den örtlichen Erhebungen um diese Untersuchung sehr verdient gemacht. Bei den Vorbereitungen in Teheran waren die Anregungen von Dr.M. BEHESCHTI, des obenerwähnten Doktoranden unseres Instituts, sehr nützlich. Auch die Provinzialbehörden in Shiraz haben sich sehr hilfsbereit gezeigt. Dabei ist besonders der Direktor der Höheren Landbauschule in Shiraz, Herr Ingenieur IZADIE, zu erwähnen, der zwei seiner Dörfer für die Untersuchung zur Verfügung stellte.

Den kaiserlich-iranischen Behörden und den beteiligten iranischen Fachleuten soll auch an dieser Stelle für ihr verständnisvolles Entgegenkommen und ihre bereitwillige Unterstützung des Forschungsvorhabens unser aufrichtiger Dank zum Ausdruck gebracht werden. Die Ergebnisse der Untersuchung, die in dieser Schrift vorgelegt werden, dürten ein bemerkenswertes Beispiel dafür sein, daß eine solche Zusammenarbeit auf dem Gebiet der agrarökonomischen Wissenschaft für beide Seiten von bedeutendem wissenschaftlichen Interesse und praktischem Nutzen sein kann.

otto SCHILLER 
I. Einleitung . . . . . . . . . . . . . . . . S. 9

1. Auswahl des Untersuchungsobjektes . . . . . . . . S. 9

2. Materialgewinnung . . . . . . . . . . S. 15

II. Die natürlichen und wirtschaftlichen Voraussetzungen . • . S. 17

1. Geographische Lage und Topographie. . . . . . . . . S. 17

2. Klima und Vegetation. . . . . . . . . . . . . S. 19

3. Verkehrslage und Verkehrsmittel . . . . . . . . S. 22

4. Wasser- und Energieversorgung . . . . . . . . . S. 23

5. Örtliche Einrichtungen, dörfliche Dienste und Versorgungsbetriebe ................ S. 25

6. Verwaltung. ................. S. 26

7. Ortsgeschichte. . . . . . . . . . . . . S. 28

8. Dorfanlage, Bauweise und Wohnverhältnisse....... S. 30

9. Dorftyp . . . . . . . . . . . . . . S. 36

III. Die Bevölkerung. . . . . . . . . . . . . . S. 36

1. Herkunft. . . . . . . . . . . . . S. 36

2. Einwohnerschaft . . . . . . . . . . . S. 37

3. Geschlecht. . . . . . . . . . . . . S. 38

4. Altersaufbau. . . . . . . . . . . . S. 38

5. Familienstand ................ S. 40

6. Haushal te . . . . . . . . . . . . . S. 42

7. Kinderzahl. . . . . . . . . . . . . . . S. 43

8. Verwandtschaftsverhältnisse . . . . . . . . . S. 44

9. Berufsstruktur. . . . . . . . . . . . . . S. 46

IV. Hygienische Verhältnisse und Gesundheitspflege . . . . . S. 48

1. Wasserversorgung. . . . . . . . . . . . . S. 48

2. Müll- und Abwässerbeseitigung . . . . . . . . . S. 49

3. Bestattungswesen. . . . . . . . . . . . s. 50

4. Körperpflege. . . . . . . . . . . . . . . S. 50

5. Kleidung. . . . . . . . . . . . . . . S. 51

6. Ernährung .................. S. 53

7. Geburtshilfe. . . . . . . . . . . . . . S. 56

8. Ärztlicher Dienst und klinische Betreuung . . . . . . S. 56

9. Gesundheitszustand. . . . . . . ..... S. 58

V. Erziehung und Bildung, Erholung und Umweltkontakte . . . . S. 61

1. Die Familie . . . . . . . . . . . . . . . S. 61

2. Die Dorfgemeinschaft. . . . . . . . . . . S. 62

3. Die Kirche...................... S. 62

4. Die Schule.................. S. 63

5. Bildungsstand ..................... s. 65

6. Massenkommunikationsmittel. . . . . . . . . . S. 66

7. Erholung und Feste. . . . . . . . . . . . S. 66

8. Umweltkontakte. . . . . . . . . . . . . S. 69

9. Politische Betätigung. . . . . . . . . . . . S. 70 
VI. Die Landbewirtschaftung . . . . . . . . . . S. 70

1. Besitz- und Eigentumsverhältnisse. . . . . . . S. 70

2. Pachtverhältnisse. . . . . . . . . . . . S. 74

3. Bodennutzung . . . . . . . . . . . . . . S. 82

4. Arbeitsverfassung, Arbeitsmethoden und Arbeitsgeräte . . S. 85

VII. Die Viehwirtschaft. . . . . . . . . . . . . S. 89

1. Viehzucht und Vieherwerb . . . . . . . . . S. 89

2. Viehhaltung. . . . . . . . . . . . . . . S. 90

3. Fütterung. . . . . . . . . . . . . . . . S. 91

4. Nutzungsrichtung urd Leistungen. . . . . . . . S. 92

VIII. Die wirtschaftlichen Verhältnisse . . . . . . . . S. 94

1. Vermögensverhältnisse. . . . . . . . . . . S. 94

2. Einkommen. . . . . . . . . . . . . . . S. 97

3. Verschuldung .................... S. 99

IX. Das Entwicklungsprogramm. . . . . . . . . . . S. 101

X. Schlußbetrachtung . . . . . . . . . . S. 106

A $n$ h a $n g$ : . . . . . . . . . . . . . . . . S. 119

Anlage 1: Dorf-Erhebungsbogen . . . . . . . . . . . S. 121

Anlage 2: Haushalts- und Betriebsbogen. . . . . . . . S. 126

Anlage 3: Bilder aus den Untersuchungsdörfern . . . . . . S. 127

Literaturverzeichnis. . . . . . . . . . . . . . S 131 
Verzeichnis der Übersichten und Abbildungen

Übersicht 1: Wochenspeiseplan eines Dorfbewohners . . . . . . S. 55

" 2: Zahl und Art der Krankheitsfälle in Zarqan 1959/60 . S. 60

" 3: Aufteilung der Getreideernte 1960 bei vier ausgewählten Pächtergemeinschaften. . . . . . . . S. 80

11

4: Das Jahreseinkommen einer Pächterfamilie, 1960 . . . S. 97

Abbildung 1: Mittlere jährliche Niederschläge im Iran . . . . S. 10

2: Lageskizze der Untersuchungsdörfer in der Ramjird-

Ebene. . . . . . . . . . . . . S. 18

3: Klimadiagramm von Shiraz .......... S. 20

4: Die Gliederung der Verwaltung in der Provinz Fars. . S. 27

5: GrundriB des Dorfes Gondzereschk / Amt Ramjird . . S. 31

6: GrundriB des Dorfes Mansur-abad / Amt Ramjird. . . S. 31

7: Hof eines Bauern und seiner beiden verheirateten

Söhne.................. S. 33

8: Altersaufbau der Einwohner von G. und M. . . . . . S. 40

9: Verwandtschaftsverhältnisse zwischen den Haushalts-

vorständen . . . . . . . . . . . . S. 45

10: Gemarkung Mansur-abad und Gondzereschk/Amt Ramjird. S. 73

11: Plan der Neuanlage des Dorfes G. (Gondzereschk). . . S. 104

12: Plan eines neuen Gehöftes in G. (Gondzereschk) . . . S. 104 


\section{Anmerkungen}

Da eine verbindliche Umschrift der iranischen Vokalisation fehlt, wurde die Schreibweise der iranischen Worte möglichst der deutschen Sprache angepabt.

Die Schreibweise der iranischen Ortsnamen entspricht jedoch, soweit festgestellt werden konnte, der Schreibweise in "Goldmanns GroBer Weltatlas", Miinchen 1955.

Alle Geldwerte wurden des besseren Verständnisses wegen in Deutsche Mark umgerechnet, wobei 20 Rials $=1$ DM bewertet wurden.

Die hinter den Verfassernamen in [...] angegebenen Ziffern beziehen sich auf das im Literaturverzeichnis unter dieser Ziffer aufgefürte Werk. Untersuchungen über den Teilbau im Iran, die nach AbschluB der vorliegenden Dorfmonographie durchgeführt wurden, trugen zu einer weiteren Klärung der Begriffe bei. Danach wird das Verhältnis von Grundherren und abhängigen Dorfbewohnern besser als Teilbauverhältnis beschrieben und werden die in der vorliegenden Studie als Pächter oder Teilpächter erwähnten Dorfbewohner genauer als "Anteilsbauern" gekennzeichnet. (Vgl. Ulrich PLANCK, Der Teilbau im Iran, Ber. ü. Landw. H. 2/1962) 